\title{
Space Applications of the FLUKA Monte-Carlo Code: Lunar and Planetary Exploration
}

\author{
Kerry Lee, ${ }^{1}$ Thomas Wilson, ${ }^{1, a}$ Neal Zapp, ${ }^{1}$ and Lawrence Pinsky, ${ }^{2}$ \\ for the FLUKA collaboration ${ }^{b}$ \\ ${ }^{1}$ NASA, Johnson Space Center, \\ 2101 NASA Parkway, Code KR, Houston, Texas 77058 USA \\ ${ }^{2}$ University of Houston \\ 4800 Calhoun Blvd, Houston, Texas, 77204 USA
}

Received 15 November 2005

\begin{abstract}
NASA has recognized the need for making additional heavy-ion collision measurements at the U.S. Brookhaven National Laboratory in order to support further improvement of several particle physics transport-code models for space exploration applications. FLUKA has been identified as one of these codes and we will review the nature and status of this investigation as it relates to high-energy heavy-ion physics.
\end{abstract}

Keywords: Heavy-ion collisions, cosmic rays, particle albedos PACS: 21.60.Ka, 24.10.Lx

\section{Introduction}

The particle radiation environments on planetary surfaces contain a full spectrum of particle types, including relativistic nuclei produced in heavy-ion collisions created by incident high-energy cosmic rays. Unlike the Earth which is protected by an atmosphere, the surface of the Moon is an example of a planetary surface directly bombarded by energetic nuclei from both the Sun and the Galaxy.

As a consequence, the lunar environment is a complex one involving the full range of heavy-ion physics from thermal neutrons to interactions predicted by the relativistic Dual Parton Model (DPM). Backscatter albedos of all types are generated, including prompt neutrino production due to charmed meson decay. Defining these radiation environments is a circumstance very similar to that existing at particle accelerators such as the forth-coming Large Hadron Collider (LHC) at CERN and similar colliders at Fermilab. Monte Carlos are the simulation tools used in that definition, and FLUKA [1-3] is the one of choice in this discussion. 
It has become a goal of experiments such as NASA-funded studies at the U.S. Brookhaven National Laboratory to make as many exclusive cross-section measurements as possible and feasible in the energy range $0.1-5.0 \mathrm{GeV} / \mathrm{A}$. These can enhance the Monte Carlo modelling of nuclear physics in the transition region from nonrelativistic to relativistic effects.

\section{Technical Background on FLUKA}

As a fully-integrated particle physics Monte Carlo simulation package, FLUKA has been successfully corroborated with well-established experimental physics (called benchmarking), and is known to preserve correlations within interactions as well as among particle shower components, from thermal neutrons to $\mathrm{TeV}$ energies. Its simulation represents the interaction and propagation through matter of some 60 different types of particles with high accuracy. This includes photons and electrons from $1 \mathrm{keV}$ to thousands of $\mathrm{TeV}$, charm production of neutrinos, muons of any energy, hadrons of energies up to $20 \mathrm{TeV}$ (extended to $10 \mathrm{PeV}$ by linking FLUKA with the DPMJET code discussed below), as well as all of the corresponding antiparticles and heavy ions.

\section{Modifications of FLUKA in Progress}

Since ion-ion (A-A) nuclear interactions are not yet thoroughly treated in any Monte Carlo for all energies, FLUKA is still under development. This circumstance arises because of the paucity of exclusive A-A collision data at the onset of relativistic effects. Stated differently, the Monte Carlo community is in need of a satisfactory event generator that adequately simulates collisional nuclear physics (A-A scattering) in the energy range 0.5 -to-5.0 GeV/A.

Above this energy range (0.5-to-5.0 GeV/A), the Dual Parton Model (DPMJET) is used $[4,5]$. Below that range, FLUKA employs the Intra-Nuclear Cascade (INC) and Pre-Equilibrium Approach to Nuclear Thermalization (PEANUT) models for light ions.

\subsection{The FLUKA-DPMJET Interface}

The event generator DPMJET mentioned above is based on the Dual Parton Model in conjunction with the Glauber formalism. It is a Monte Carlo code for sampling hadron-hadron, hadron-nucleus, and nucleus-nucleus (A-A) collisions at accelerator and cosmic-ray energies ( $\mathrm{E}_{l a b}$ from $5-10 \mathrm{GeV} / \mathrm{A}$ up to $10^{18}-10^{20} \mathrm{eV} / \mathrm{A}$ ), adapted and interfaced with the rest of FLUKA [4]. It has since been upgraded to DPMJET$3[5]$.

FLUKA internally requires A-A production cross-sections in order to select the appropriate nucleus-nucleus interactions. It includes a complete matrix of A-A production cross-sections along with the Glauber impact parameter distributions. 
Owing to the validity of the Glauber formalism, these cross-sections can be safely utilized down to a projectile kinetic energy of about $1 \mathrm{GeV} / \mathrm{A}$.

\subsection{Transition to Non-relativistic Energies}

Although event generators exist for most of the particle interactions treated by FLUKA, those necessary for A-A collisions in the transition region from the Glauber formalism down to non-relativistic energies (from say $5 \mathrm{GeV} / \mathrm{A}$ down to $100 \mathrm{MeV} / \mathrm{A}$ ) are not as well-defined. This is the region of the NASA-funded transport code investigation.

One feature of the physics in this region is momentum-dependent nuclear potentials which are notoriously nonlocal in nature. Nonlocal interactions break Lorentz invariance and this is somehow related to the transition to nonrelativistic dynamics (where Lorentz invariance becomes negligible). A number of computer codes have been created to deal with the nuclear and molecular dynamics in this energy range, but with only marginal success. The first was the nonrelativistic Quantum Molecular Dynamics (QMD) model [6], augmented by limited Poincaré invariance arguments to be pseudo-relativistic, a model known as the Relativistic QMD (RQMD) method [7].

The RQMD 2.4 model has been widely used over the energy range from 0.1 $\mathrm{GeV} / \mathrm{A}$ to several hundreds of $\mathrm{GeV} / \mathrm{A}$. However, this code does not perform the latest stages of the A-A reaction, and does not always conserve energy.

Several alternatives exist, addressed in more detail elsewhere [8]. First, the RQMD code per se has been scrutinized, adapted to be interfaced with the FLUKA evaporation models and corrected for energy non-conservation, while maintaining its underlying philosophy. Then it has been embedded into the current version of FLUKA while again guaranteeing absolute conservation of energy, an essential requirement for any suitable transport code. Second, alternative QMD [3] and Hamiltonian Molecular Dynamics (HMD) event generators are undergoing development.

\subsection{The Modified RQMD Event Generator}

The modified RQMD event generator included in the present release as RQMDFLUKA has been used to prepare fluence plots that aided in planning for the proposed measurements at Brookhaven National Laboratory for NASA. These are of interest, and two will be shown here while more are discussed in a different publication [8]. They illustrate FLUKA's existing capability in the suspect energy range below DPMJET's cutoff around $5 \mathrm{GeV} / \mathrm{A}$ and above the INC-technique and PEANUT.

The embedded RQMD-FLUKA provides an option to run in an INC-like mode to reduce computation time in instances where full accuracy is not required. The original RQMD code does not identify complex fragments at the end of the interaction. The strategy adopted is to allow the code to follow an interaction to 
some intermediate point where all of the hard collisions between constituent particles are over. Then the evolution is stopped in an interim state. At that point, projectile- and target-like nuclei are formed out of the spectator nucleons (i.e. nucleons which did not undergo two-body collisions), and their excitation energy is calculated from the energies of the holes left by the hit nucleons. The remaining particles are next placed in the final-state RQMD-FLUKA event-generator output buffer. The two trial hot-fragments so identified are next run through the FLUKA evaporation/fission/fragmentation module, allowing them to de-excite into surviving fragments since the latter may have boiled-off some of their original constituents in the evaporation process.

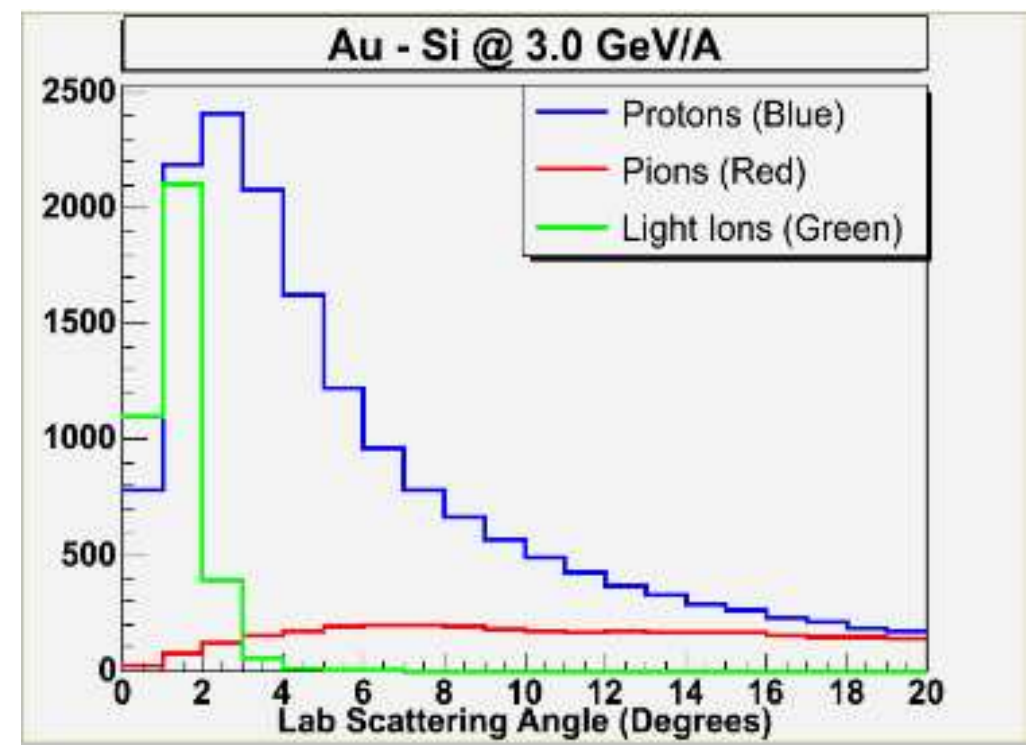

Fig. 1. Laboratory scattering angle distributions for protons, pions and light ions (D-B) per second in one-degree annular bins from a $1 \mathrm{kHz} 3.0 \mathrm{GeV} / \mathbf{A}$ Gold $(\mathbf{A u})$ beam incident on a Silicon $(\mathbf{S i})$ target.

Figures 1-2 show some results from the RQMD-FLUKA event generator as it presently exists. These fluence plots (ordinate: number of particles $\mathrm{cm}^{-2}$ ) depict the laboratory scattering angles in annular angle-bins of one degree for protons above a kinetic energy of $100 \mathrm{MeV}$ in the laboratory, for all charged pions above $50 \mathrm{MeV}$ in the laboratory and for all light ions (Deuterons through Boron) above $100 \mathrm{MeV} / \mathrm{A}$. The ordinates are normalized to the yields from a 1-kHz beam of Gold $(\mathrm{Au})$ incident upon a one-interaction-length thick Silicon $(\mathrm{Si})$ target.

Both of the figures show a similar behavior, having a proton distribution peaking near zero. One of the important differences in the calculations for $5 \mathrm{GeV} / \mathrm{A}$ with respect to $3 \mathrm{GeV} / \mathrm{A}$ interactions is the relative height of the pion distribution at larger angles. The pion scattering-angle distribution rises from low values at small 
angles, becoming comparable to the proton fluence. In Figure 2 it begins to exceed the proton fluence around 13 degrees.

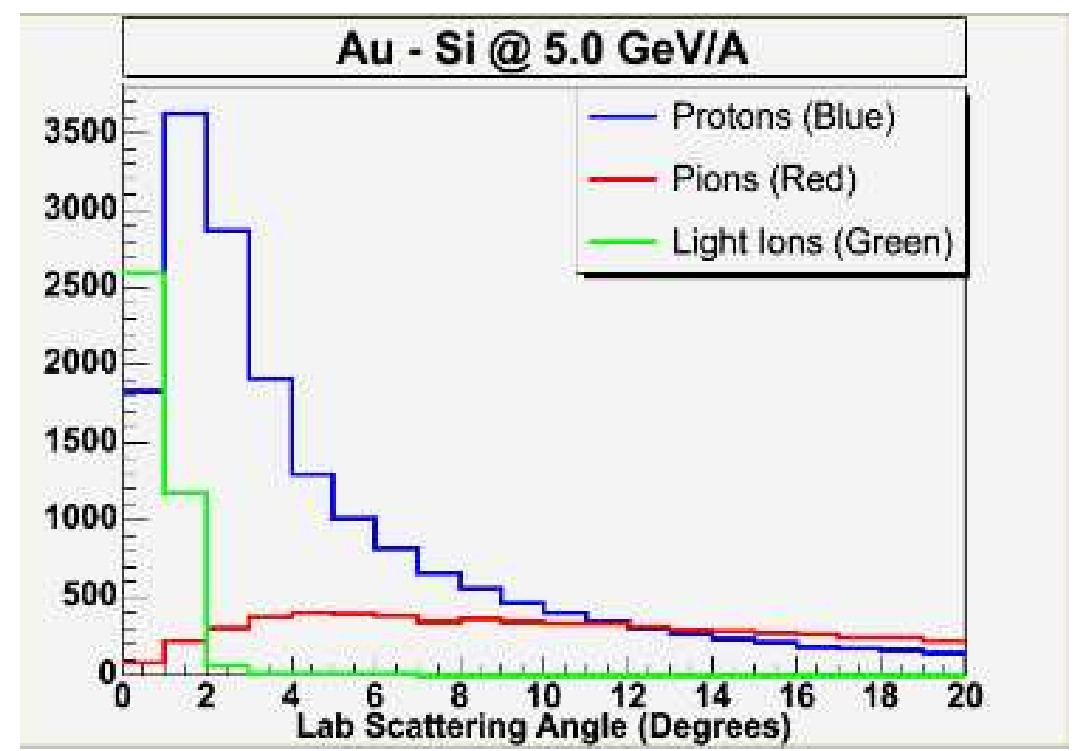

Fig. 2. Same fluence as Figure 1, except using a $1 \mathrm{kHz} \mathbf{5 . 0} \mathbf{~ G e V} / \mathbf{A}$ Gold ( $\mathbf{A u})$ beam incident on a Silicon (Si) target. Note the dominance of the pions starting at about 13 degrees.

\subsection{The HMD Event Generator}

The additional option under study to complement the RQMD-FLUKA event generator is the HMD model. This event generator derives from the concept of primary and secondary constraints developed by Dirac [9] for relativistic Hamiltonians and derived from Poincaré invariance. When the Hamiltionian exists, this approach to relativistic QMD-type methods has never been implemented successfully in a Monte Carlo and it offers promise in the problematic transition energy range. Its potential application within FLUKA is under consideration as yet another alternative to QMD-FLUKA. More detail on this method is published elsewhere [8].

\section{Conclusions}

Prompted by the need for adequate Monte Carlo simulation tools both for future particle accelerators and space exploration architectures, FLUKA is undergoing continuing development. The need for better understanding of heavy-ion physics in the transition from nonrelativistic to relativistic energies has been reviewed. 


\section{Acknowledgments}

FLUKA development as discussed here has been partially funded by financial support from INFN as well as NASA under Grant NAG8-1901.

\section{Notes}

a. Permanent address: NASA, Johnson Space Center - KR, Houston, Texas 77058; E-mail: twilson@ems.jsc.nasa.gov

b. V. Andersen ${ }^{1}$, F. Ballarini ${ }^{2,3}$, G. Battistoni ${ }^{3,4}$, M. Campanella ${ }^{3,4}$, M. Carboni $^{5}$, F. Cerutti $^{3,4}$, N. Elkhayari ${ }^{1}$, A. Empl ${ }^{1}$, A. Fassò ${ }^{6}$, A. Ferrari ${ }^{7}$, E. Gadioli ${ }^{3,4}$, M. V. Garzelli $^{3,4}$, M. LeBourgeois ${ }^{1}$, K. T. Lee ${ }^{1,8}$, B. Mayes $^{1}$, S. Muraro ${ }^{3}$, A. Ottolenghi ${ }^{2,3}$, M. Pelliccioni ${ }^{5}$, L. S. Pinsky ${ }^{1}$, T. Rancati ${ }^{3}$, J. Ranft $^{9}$, S . Roesler ${ }^{7}$, P. R. Sala ${ }^{3,4}$, D. Scannocchio $^{2,3}$, G. Smirnov ${ }^{10}$, V. Vlachoudis ${ }^{7}$, T. Wilson ${ }^{8}$, and N. Zapp ${ }^{1,8}$

${ }^{1}$ University of Houston, Houston, TX, USA

${ }^{2}$ University of Pavia, Milan, Italy

${ }^{3}$ Istituto Nazionale di Fisica Nucleare (INFN), Milan, Italy

${ }^{4}$ University of Milan, Milan, Italy

${ }^{5}$ Laboratori Nazionali di Frascati, INFN, Milan, Italy

${ }^{6}$ Stanford Linear Acclerator Center (SLAC), Stanford, CA, USA

${ }^{7}$ European Organization for Nuclear Research (CERN), Geneve, Switzerland

${ }^{8}$ NASA Johnson Space Center, Houston, TX, USA

9 Siegen University, Siegen, Germany

${ }^{10}$ Joint Institute for Nuclear Research (JINR), Dubna, Russia

\section{References}

1. A. Fassò, A. Ferrari and P. Sala, Proceedings of Monte Carlo 2000 Conf., Lisbon, October 23-26, 2000, A. Kling, F. Barao, M. Nakagawa, L. Tavora, P.Vaz, eds., Springer-Verlag, Berlin (2001) p. 159.

2. A. Fassò, A. Ferrari, J. Ranft and P. Sala, ibid., p. 955.

3. V. Andersen et al., Adv. Spa. Res. 34, Issue 6 (2004) 1302.

4. J. Ranft, Phys. Rev. D51 (1995) 64.

5. S. Roesler, R. Engel and J. Ranft, Proceedings of Monte Carlo 2000 Conf., Lisbon, October 23-26, 2000, Springer-Verlag, Berlin (2001) p. 1033.

6. J. Aichelin, Prog. Part. Nucl. Phys. 30 (1993) 191.

7. H. Sorge, H. Stöcker and W. Greiner, Ann. Phys. 192 (1989) 266.

8. L. S. Pinsky et al., IEEE Aerospace Conf., Big Sky, Montana, to be published (2005).

9. P. A. M. Dirac, Rev. Mod. Phys. 21 (1949) 392. 